\title{
Transatlantica
}

Revue d'études américaines. American Studies Journal

\section{Keith Booker (ed.), Encyclopedia of Comic Books and Graphic Novels 2 vols., Greenwood Press, 2010}

Jean-Paul Gabilliet

\section{(2) OpenEdition}

\section{Journals}

Édition électronique

URL : https://journals.openedition.org/transatlantica/4967

DOI : $10.4000 /$ transatlantica.4967

ISSN : 1765-2766

Éditeur

Association française d'Etudes Américaines (AFEA)

Référence électronique

Jean-Paul Gabilliet, « M. Keith Booker (ed.), Encyclopedia of Comic Books and Graphic Novels 2 vols. Greenwood Press, 2010 », Transatlantica [En ligne], 1 | 2010, mis en ligne le 27 septembre 2010, consulté le 10 février 2023. URL : http://journals.openedition.org/transatlantica/4967 ; DOI : https:// doi.org/10.4000/transatlantica.4967

Ce document a été généré automatiquement le 10 février 2023.

Creative Commons - Attribution - Pas d'Utilisation Commerciale - Pas de Modification 4.0 International - CC BY-NC-ND 4.0

https://creativecommons.org/licenses/by-nc-nd/4.0/ 


\title{
M. Keith Booker (ed.), Encyclopedia of Comic Books and Graphic Novels 2 vols., Greenwood Press, 2010
}

\author{
Jean-Paul Gabilliet
}

1 Les encyclopédies thématiques éditées par Greenwood Press sont des ouvrages à destination des bibliothèques (comme l'indique leur prix toujours élevé). Leur vocation principale est d'être consultées par des étudiants, des chercheurs novices sur une question donnée, voire de fournir des aide-mémoire à des chercheurs confirmés (mission qu'elles remplissaient fort bien longtemps avant l'apparition de Wikipedia). Sans viser à l'exhaustivité, ces ouvrages ambitionnent de proposer des panoramas représentatifs de domaines précis.

2 Réalisés sous la direction de M. Keith Booker, Professeur au département d'anglais de l'Université d'Arkansas, les deux volumes formant l'Encyclopedia of Comic Books and Graphic Novels rassemblent sur 763 pages plus de 330 entrées rédigées par quatre-vingts auteurs. Présentées par ordre alphabétique, celles-ci se divisent en cinq catégories: Individual Comic Book and Graphic Novel Titles or Characters (180), Individual Writers and Artists (103), Themes and Genres (31), Comics Publishers (11), Miscellaneous (8). Ces chiffres semblent bien minces pour couvrir un secteur de l'édition vieux de trois-quarts de siècle et ayant produit au moins 170000 comic books et plusieurs centaines de graphic novels mais, comme toujours en histoire culturelle, le quantitatif ne doit pas occulter le qualitatif. Sous un titre faussement englobant, l'ouvrage de Booker vise à présenter les éléments d'un certain canon de la bande dessinée déterminé dans le temps et l'espace. L'espace est l'ensemble nord-américain, sur les marges duquel se greffent quelques noms européens et japonais. Le temps, sur les trois quarts de siècle abordés, se concentre très fortement sur les vingt dernières années, essentiellement en raison de l'attention portée aux graphic novels, support commercial et forme d'expression ayant progressivement supplanté les traditionnels comic books (il y a fort à parier que d'ici une dizaine d'années, ce terme aura pris en anglais les mêmes connotations surannées que « revues illustrées » en français). À l'évidence, l'ouvrage a été clairement conçu pour 
être utilisé dans le contexte de l'augmentation permanente du nombre de cours consacrés à la bande dessinée dispensés dans les universités nord-américaines. Mais, précaution oratoire indispensable, qui dit graphic novels ne dit pas nécessairement comic books : une des phrases les plus fréquemment entendues de nos jours sur les campus est «I don't read comic books-I only read graphic novels. »

Pour des Français familiarisés depuis les années 60 avec le processus de progressive respectabilisation de la bande dessinée qu'a connu notre pays, une affirmation pareille semble saugrenue. Elle recouvre néanmoins une réalité tout à fait tangible, à savoir le mouvement ascendant du moyen d'expression "bande dessinée » dans les hiérarchies culturelles nord-américaines depuis la fin des années 80 , grâce à l'émergence d'un support qui a fait basculer la BD du domaine de la presse enfantine-adolescente à celui du livre, grosso modo vingt ans après qu'un phénomène semblable avait eu lieu en Europe occidentale. Le graphic novel, équivalent approximatif de nos "albums" de bande dessinée, constitue depuis le début du $\mathrm{xxI}^{\mathrm{e}}$ siècle un des nouveaux horizons des humanités (principalement en littérature, communication, philosophie et études cinématographiques, dont les comics studies sont en passe de devenir un adjuvant de plus en plus fréquent). Cependant, le corpus que favorisent de fait des enseignants majoritairement formés à la critique littéraire, cinématographique, esthétique plutôt qu'à l'analyse historique est celui de la bande dessinée d'auteur publiée depuis une vingtaine d'années sous forme de graphic novels, la production du demi-siècle antérieur se prêtant moins bien à l'application d'outils critiques conçus à l'origine pour disséquer des productions d'auteur plutôt que de la littérature de masse.

Une autre inflexion non dite de cette encyclopédie, peut-être du fait de l'aire de spécialisation initiale de son directeur, est son tropisme cinématographique. Celui-ci a des effets qu'il convient de souligner: proposer en illustration d'un ouvrage de référence destiné à être beaucoup consulté par des novices plus d'une trentaine de photographies tirées d'adaptations cinématographiques et télévisuelles de bandes dessinées au lieu d'images dessinées constitue un choix éditorial surprenant - celui-ci se justifie probablement par des questions de droits mais il pose de fait une permanente équivalence floue entre images de cinéma et images de bande dessinée qui dilue implicitement la spécificité de ces dernières. À un niveau autre, il y a lieu de s'interroger sur la pertinence d'utiliser des photos venues de films reniés par les auteurs des bandes dessinées originales (comme c'est le cas pour Fritz the Cat de Robert Crumb, From Hell d'Alan Moore et Eddie Campbell, The League of Extraordinary Gentlemen d'Alan Moore et Kevin O'Neill ou Watchmen d'Alan Moore et Dave Gibbons) ou encore sur le choix de mettre en avant des graphic novels dont la fortune critique tient plus à l'adaptation pour le grand écran qu'à la réception critique initiale - passe encore que des entrées soient consacrées à The History of Violence (D. Cronenberg, 2005) et The Road to Perdition (S. Mendes, 2002) mais le lecteur aimerait trouver les arguments démontrant que Mystery Men (K. Usher, 1999) ne fut pasun échec par rapport au comic book décalé de Bob Burden dont il était tiré. Et pourquoi, dès lors, ne pas avoir consacré des entrées à The Mask (C. Russell, 1994) et Men in Black (B. Sonnenfeld, 1997 et 2002), exemples d'adaptations fort réussies de bandes dessinées inégales? C'est une des grosses lacunes de cette encyclopédie que l'absence de réflexion sur les liens bande dessinée-cinéma, alors même qu'une des entrées synthétiques est titrée "Adaptations from other media.» 
5 Le titre à deux étages de cette encyclopédie (comic books and graphic novels) manifeste assez clairement l'équilibre instable, à cheval sur deux traditions culturelles qui caractérise l'utilisation de bande dessinée dans les cursus universitaires nordaméricains aujourd'hui. La volonté de mettre sur le même plan, d'une part, un corpus de graphic novels récents entretenant des homologies fortes avec la production littéraire et/ou cinématographique d'auteur, et par là même tout à fait adaptés à la mise en œuvre d'un discours critique esthétique, et d'autre part, une sélection d'entrées consacrées à des auteurs et séries grand public caractéristiques de ce que fut l'industrie des comic books pendant ses cinquante premières années, manifeste un œcuménisme louable. Mais le désir de présenter un panorama aussi large que possible a un revers : la représentativité du corpus des entrées relatives aux graphic novels est très supérieure à celle des entrées relatives aux comic books. Cette sensation de déséquilibre est assez embarrassante pour les entrées concernant des créateurs de comic books: si l'impossibilité d'exhaustivité excuse partiellement l'absence de "grands noms" (encore qu'on voit difficilement quelle raison autre que le nombre de signes pourrait justifier l'omission du dessinateur John Buscema, du scénariste-editor Archie Goodwin, des auteurs qui firent la gloire de l'éditeur EC Comics après la guerre - Johnny Craig, George Evans, Graham Ingels, Bernard Krigstein - ou encore des créateurs underground parmi les plus emblématiques tels que Vaughn Bodé, Rory Hayes ou Greg Irons), elle justifie moins la mise en avant de créateurs dont l'apport durable à la bande dessinée américaine reste à démontrer (Chuck Dixon, Steve Leialoha, Fabian Nicieza, Joss Whedon par exemple). Encore plus gênant, à ce niveau, est le fait d'avoir uniformisé la longueur des entrées consacrées à des auteurs individuels : consacrer autant de signes à John Severin ou sa sœur Marie Severin, artisans honorables mais sans génie de l'édition grand public, qu'à des créateurs qui ont influencé profondément les générations qui les ont suivis, tel Will Eisner, Jack Kirby ou Harvey Kurtzman, apparait comme un choix difficilement justifiable. Dans les trois cas précités, l'apport d'acteurs hors du commun au champ créatif concerné se trouve minimisé de manière impardonnable.

Une autre fausse bonne idée est le désir de ne pas exclure la production étrangère. On se prend à regretter que l'encyclopédie ne se soit pas franchement et clairement centrée sur l'Amérique du nord (ou plutôt les États-Unis, car le Canada n'est évoqué qu'au travers des entrées consacrées à Chester Brown, Seth et Dave Sim et à leurs œuvres). Deux auteurs européens (Hergé et Moebius) et un Japonais (Tezuka) ainsi qu'une demi-douzaine de séries font l'objet de notices individuelles. Quant à l'entrée «European Comics» rédigée par le spécialiste belge Pascal Lefèvre, elle illustre la difficulté extrême qu'il y a à postuler un objet d'analyse ou d'histoire tel que « la bande dessinée européenne ", la diversité des traditions nationales ne permettant de dégager que des analogies de surface sur la longue durée (et, reconnaissons-le, Lefèvre réalise probablement la meilleure synthèse possible sur ce sujet patchwork dans la limite du nombre de signes qui lui est imparti !). Cette entrée démontre par ailleurs le caractère lacunaire de l'index qui omet plusieurs dizaines de noms et titres cités dans les entrées synthétiques.

7 On ne se livrera pas ici à un fastidieux recensement des erreurs et imprécisions rencontrées au cours de la lecture, essentiellement parce que leur nombre est finalement assez bas quand on le rapporte à la masse de données brassée dans cette encyclopédie. Tout au plus pourra-t-on déplorer la qualité inégale des entrées. Certaines, notamment dans les articles synthétiques, proposent de bons résumés 
constituant des points de départ fiables pour un travail de recherche tandis que d'autres se situent à un niveau de généralité qui n'apporte pas grand chose. Il en va de même pour les références bibliographiques en fin d'entrées, dont le spectre va de listes d'ouvrages fiables à des pages web ou des références imprimées que l'on qualifiera par charité d'anecdotiques (par exemple, l'entrée consacrée au dessinateur Bill Sienkiewicz donne pour unique référence bibliographique une interview de trois pages publiée en 1985 dans Marvel Age, mensuel auto-promotionnel édité à l'époque par Marvel).

En conclusion, l'Encyclopedia of Comic Books and Graphic Novels est un produit fini assez décevant (et l'auteur de ces lignes est le premier à le déplorer). Néanmoins, en l'absence de tout ouvrage équivalent sur le domaine nord-américain, il est dommage de s'en priver comme un outil de travail préliminaire dans une recherche portant sur les comic books et le roman graphique, en dépit de son index largement inutilisable. Malgré ses défauts, elle donne une image assez claire du "canon » le plus contemporain dans lequel se reconnaissent les universitaires nord-américains étudiant et enseignant le graphic novel sous l'angle littéraire et esthétique au début du XxI ${ }^{e}$ siècle. Son utilité est beaucoup plus limitée pour une recherche de nature historique embrassant la totalité de l'édition de comic books depuis les années 30. On ne peut que souhaiter que Greenwood ou un autre éditeur propose un jour un ouvrage de référence fiable consacré à ce qu'étaient les comic books avant qu'ils ne devinssent de respectables graphic novels.

INDEX

Thèmes : Recensions

\section{AUTEUR}

JEAN-PAUL GABILLIET

Université Bordeaux III 